\title{
Paratesticular Tumors: A Report of Two Rare Cases
}

\author{
Sinchana K.M., Meena N. Jadhav*, Rujutha Datar and Shreekant K. Kittur \\ Department of Pathology, Belagavi Institute of Medical Sciences, Belagavi, India
}

\begin{abstract}
Paratesticular tumors are rare comprising $7-10 \%$ of total intrascrotal tumors of which $70 \%$ are benign and $30 \%$ are malignant. Leiomyomas and undifferentiated pleomorphic sarcomas are two such rare paratesticular tumors which should be accurately diagnosed for purpose of treatment and prognosis. We report here two cases. In first case, a 65-year-old man presented with paratesticular mass of 12 years duration. Histology showed features of benign spindle cell tumor. On immunohistochemistry the tumor cells were positive for vimentin, desmin, h-caldesmon and SMA. Based on these findings a diagnosis of leiomyoma of scrotum was made. In second case, a 66-year-old male presented with left paratesticular mass of eight months duration. Histology showed features of high-grade sarcoma. On immunohistochemistry tumor cells were focally positive for SMA and negative for h-caldesmon, desmin, calretinin and ALK-1. A final diagnosis of undifferentiated pleomorphic sarcoma of paratesticular region, grade $3 / 3$ was made.
\end{abstract}

Keywords: Leiomyoma, Scrotum, Testis, Undifferentiated Pleomorphic Sarcoma

\section{Introduction}

Paratesticular tumors are rare comprising $7-10 \%$ of total intrascrotal tumors of which $70 \%$ are benign and $30 \%$ are malignant. ${ }^{[1]}$ Leiomyoma and undifferentiated pleomorphic sarcoma are two such rare tumors which should be accurately diagnosed for purpose of treatment and prognosis. Scrotal leiomyomas are essentially benign arising from the subcutaneous tissue or dartos muscle. ${ }^{[2]}$ Recurrence and malignant transformation to leiomyosarcoma have been reported. ${ }^{[3]}$ Undifferentiated pleomorphic sarcoma (UPS) usually arises from soft tissues of extremities or trunk and sometimes in retroperitoneum. A limited number of cases of undifferentiated pleomorphic sarcoma originating from spermatic cord have been reported in the literature. ${ }^{[4]}$ We report here two cases of scrotal leiomyoma and undifferentiated pleomorphic sarcoma which posed diagnostic problem.

\section{Case Report}

Case 1: A 65-year-old man presented with pedunculated ulcerated mass in midline of scrotum of 12 years duration. On examination the mass felt separate from testis. Bilateral testes were normal and there were no palpable inguinal lymph nodes. It was clinically diagnosed as infected papilloma of scrotum. The excised swelling measured $8 \times 5 \times 5 \mathrm{cms}$. There was an ulcer over the skin which measured $3 \mathrm{~cm}$ in diameter filled with necrotic material. Cut surface showed circumscribed tumor with solid, pale white to yellowish areas and whorled appearance (Figure:1a \& 1b).
Microscopic examination showed ulcerated squamous epithelium, underlying dartos muscle and a partly capsulated tumor composed of ovoid to spindle cells arranged in bundles, fascicles and storiform pattern in the background of edematous stroma. These cells showed elongated vesicular nuclei with blunt to pointed ends. The nuclear chromatin was fine and showed prominent nucleoli at places. Mitosis were less than 1/10 high power field. Areas of calcification were seen in the periphery of tumor. The blood vessels were dilated with hyalinization at places. There was perivascular lymphoplasmacytic infiltration. The resected margin showed presence of tumor (Figure:1c \& 1d). A diagnosis of benign spindle cell tumor suggestive of inflammatory myofibroblastic tumor was made. On immunohistochemistry the neoplastic spindle cells expressed vimentin, desmin, h-caldesmon and smooth muscle actin(SMA)(Figure:2). Ki-67(mib-1) proliferative index was $<5 \%$. Based on these findings a diagnosis of leiomyoma of scrotum was made. The patient is on regular follow up and there is no recurrence of tumor.

Case 2: A 66 year man presented with left sided painless scrotal mass of 8 months duration. There was no history of anorexia, weight loss, malaise and pain in the swelling. Local examination revealed a hard left paratesticular mass measuring $5 \times 4 \mathrm{cms}$ which felt seperately from testis, epididymis and spermatic cord. Inguinal lymph nodes were not palpable. On ultrasonography a heterogenous lesion was noted along the course of spermatic cord on left side which measured $3.1 \times 1.8 \mathrm{~cm}$ with few hypoechoeic areas and septations. CT scan of abdomen and thorax was 
normal. Blood investigations were done: $\mathrm{Hb}-9 \mathrm{gm} / \mathrm{dl}$, WBC - 9200 cells/cumm, ESR-30mm at the end of 1 hour and Random blood sugar $-123 \mathrm{mg} / \mathrm{dl}$. Serum AFP, Beta-hCG and $\mathrm{LDH}$ were within normal limits. A clinical diagnosis of left sided paratesticular tumor was made. On FNAC the aspirate was paucicellular and inadequate for opinion. Orchidectomy was done along with excision of mass. The excised specimen showed mass with attached spermatic cord. The spermatic cord measured $7.5 \mathrm{~cm}$ in length. Outer surface of mass was nodular. Cut surface showed well circumscribed multilobulated mass seen above the testis measuring $7 \times 6 \times 4 \mathrm{~cm}$ with pale brown to grey-white areas. Cut surface of testis was unremarkable (Figure:3a \& 3b).

Microscopy of the mass showed partly capsulated tumor composed of pleomorphic tumor cells arranged in lobules, sheets with dense lymphoplasmacytic infiltration and areas of collagenous stroma. These cells were spindle to stellate shaped with hyperchromatic nuclei and 1-4 prominent nucleoli. The nuclei showed fine to coarse chromatin with multilobulation and multinucleation. At places bizarre giant cells were seen showing abundant intense eosinophilic cytoplasm with 4-5 nuclei. Abnormal mitosis was 3-4/ high power field. Blood vessels showed hyalinization at places. There were no areas of necrosis. There were no areas showing differentiation towards adipose tissue, smooth muscle, skeletal muscle or neural tissue. Sections from spermatic cord and testis were unremarkable (Figure-3c \& 3d). A diagnosis of high grade sarcoma of paratesticular region was made. On immunohistochemistry the tumor cells showed focal immunoreactivity for SMA and were negative for h-Caldesmon, desmin, calretinin and ALK1 (Figure:4). Thus a final diagnosis of undifferentiated pleomorphic sarcoma, paratesticular region Grade 3/3 (FNCLCC grading system) was made. This patient is on regular follow up and he presented with recurrence two years after the excision of mass along with metastasis to the lung and is on chemotherapy.

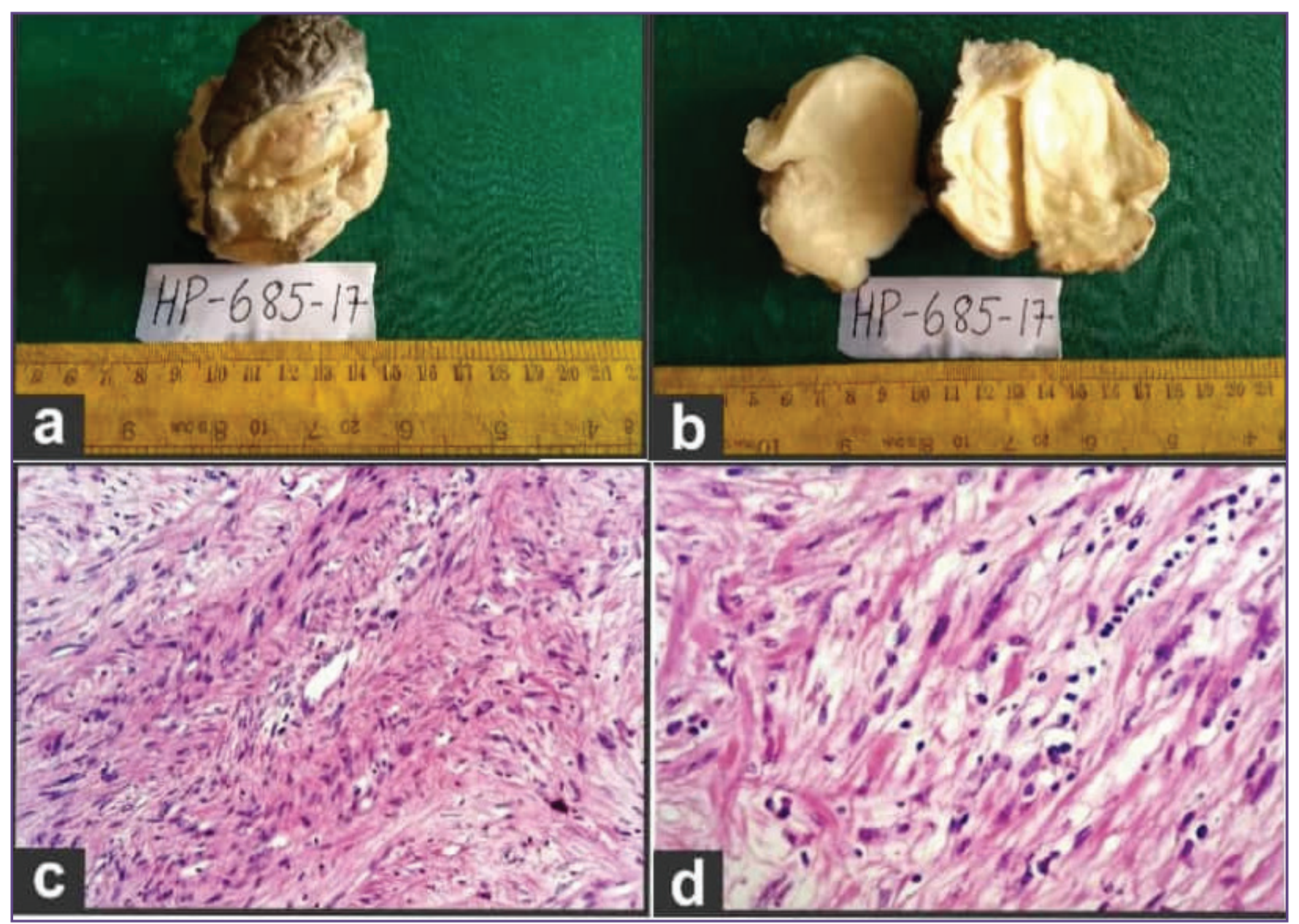

Fig. -1a: Gross specimen of skin covered mass with, B: grey white cut surface; C: Photomicrograph showing interlacing bundles of spindle cells arranged in fascicles with, D: Elongated bland nuclei (H \& E, X 400). 


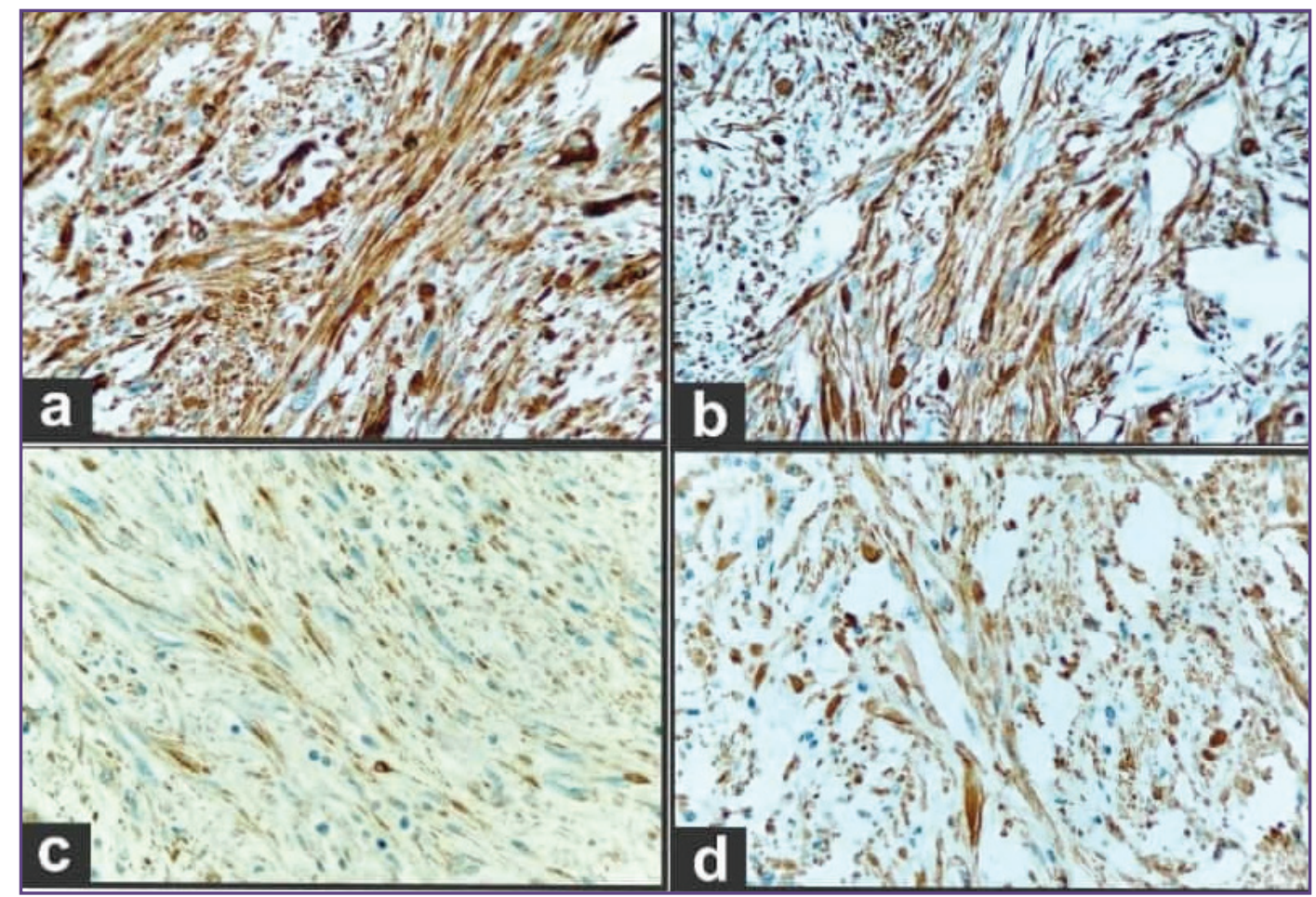

Fig. 2: Photomicrograph showing tumor cells immunoreactive for a) Vimentin; b) Desmin: C) h-Caldesmon; d) SMA (IHC,X400).
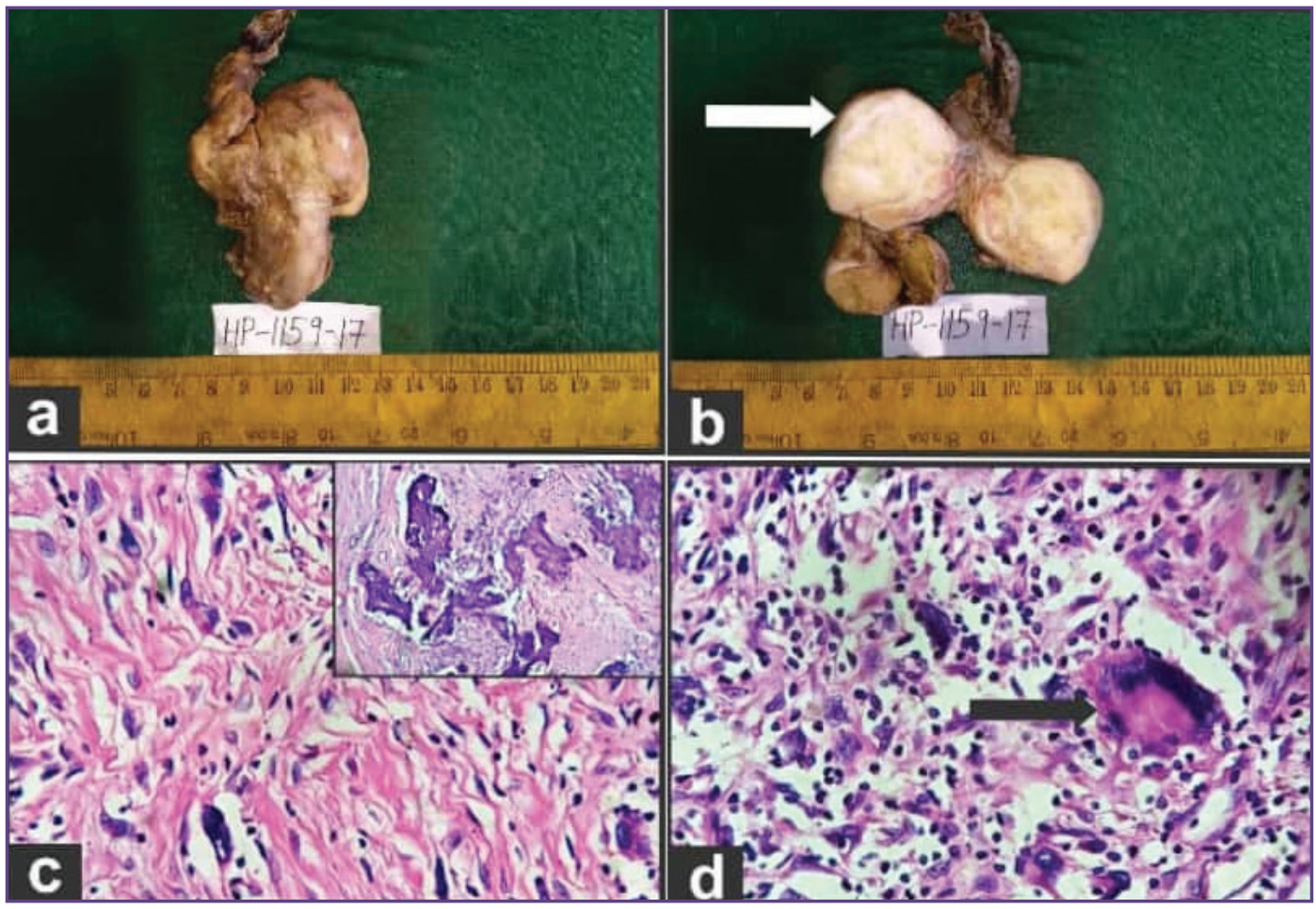

Fig. 3-a) Gross photograph of left orchidectomy specimen showing nodular mass with b) grey white cut surface; c) Photomicrograph showing pleomorphic spindle cells with metaplastic bone in the periphery (inset:H\&E,X100) and d) giant cells (H\&E,X400). 


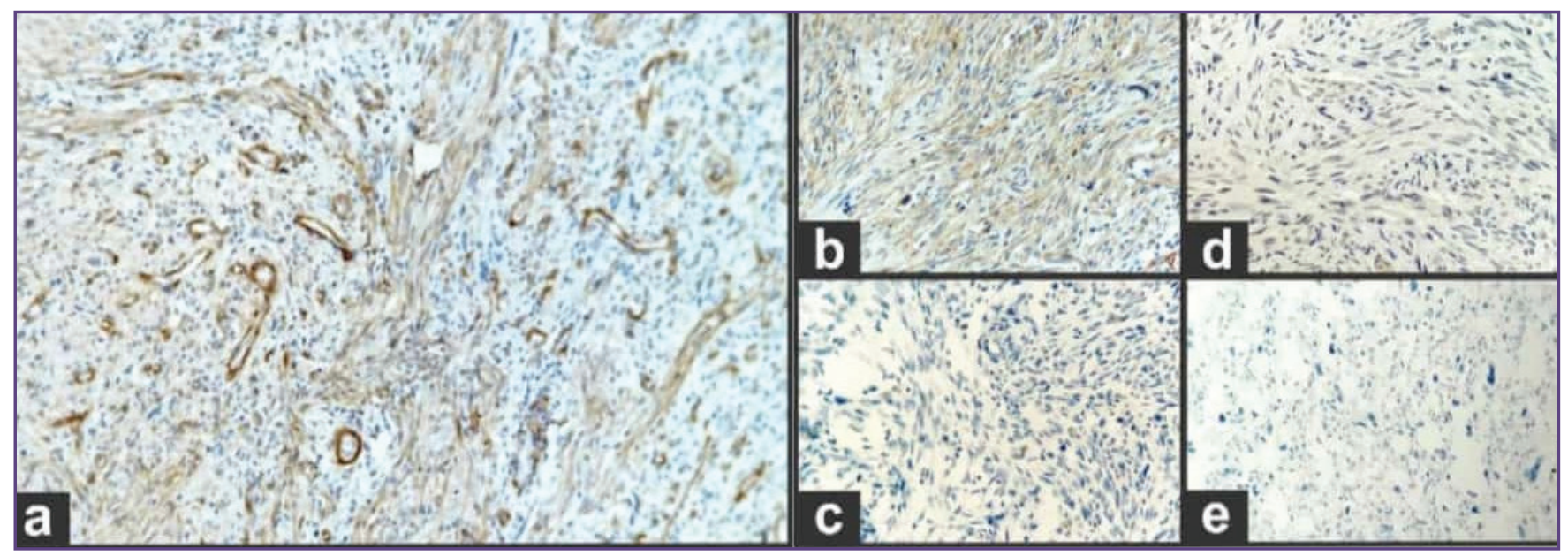

Fig. 4: Photomicrograph showing focal positivity of tumor cells for a) SMA and are negative for b) h-Caldesmon; c) Desmin: d) Calretinin; e) ALK-1 (IHC, X400).

\section{Discussion}

Scrotal leiomyoma is a rare tumor with $<50$ cases reported in the literature. It presents in $5^{\text {th }}$ decade of life. ${ }^{[3]}$ Leiomyomas are divided into three groups. The first group constitute cutaneous leiomyoma which include genital leiomyoma and involves scrotum, vulva or nipple. The second and third group of benign smooth muscle tumors includes angiomyomas and leiomyomas of deep soft tissues respectively. Scrotal leiomyoma arise from the dartos muscle and tend to be large. Patients usually present with painless, solitary small cutaneous lesion measuring 1-14 $\mathrm{cm}$ with an average size of $6.4 \mathrm{~cm}$. It has been suggested that these tumors are of myofibroblastic origin. Genital leiomyomas tend to be more cellular and they display a greater range of histologic appearances like myxoid change and an epithelioid phenotype of the cells. They tend to be focally infiltrative and often are associated with lymphoid aggregates. The presence of any mitotic activity is advocated as a criterion of potential malignancy. Simple excision is indicated and is curative for leiomyoma ${ }^{[5]}$ Recurrent and malignant transformation to leiomyosarcoma has been reported. Hence regular follow up is required. Radiation should be avoided as it may induce malignant transformation..$^{[3]}$ In our first case there was no history of radiation exposure. Microscopy did not show any abnormal mitosis, myxoid change or epithelioid appearance, but showed prominent lymphoid infiltration mimicking inflammatory myofibroblastic tumor.

Paratesticular sarcomas account for $<1 \%$ of all adult sarcomas. ${ }^{[4]}$ Pleomorphic soft tissue tumors for which a line of differentiation is debatable are presently categorized as UPS. Majority of them arise from spermatic cord and usually occurs in older adult. Some of them are radiation induced. UPS of the paratesticular region are much more rare with less than 43 reported cases till date. ${ }^{[6,7]}$ They may produce paraneoplastic syndromes like leucocytosis and hypoglycemia due to production of IL-6, IL-8, granulocyte colony-stimulating factor and insulin-like growth factor II. In our second case there was no history of hypoglycemia and total WBC count was normal.

Histopathologically UPS are classified into four types: storiform-pleomorphic, myxoid, giant cell and inflammatory with storiform type being the most common. UPS may mimic the histopathologic appearance of other sarcomas, e.g., de-differentiated and pleomorphic liposarcoma, pleomorphic leiomyosarcoma. ${ }^{[8,9]}$ Pleomorphic sarcomas have a characteristically ill defined fascicular pattern, cellular and nuclear pleomorphism, bizarre tumor cells with frequent mitoses and necrosis. In our case the other sarcomas were ruled out by the absence of differentiation towards conventional/classical area of liposarcoma, leiomyosarcoma, rhabdomyosarcoma and nerve sheath tumor. On immunohistochemical staining tumor was negative for caldesmon and hence ruled out smooth muscle differentiation. It was negative for desmin which helped to rule out smooth muscle and skeletal muscle differentiation. Calretinin marker was negative. Tumor was negative for ALK-1 which helped to exclude Inflammatory myofibroblastic tumor. UPS has no specific diagnostic markers, but can show focal positivity for CD68, S100 and vimentin and is a diagnosis of exclusion. [9] Treatment of choice is radical orchidectomy despite which local recurrence has been reported by $51 \%$ cases. ${ }^{[10]}$ Both local recurrence and distant metastasis often develop within 12 to 24 months of diagnosis. Only a minority of patient develop metastasis after 5 years. In our second case patient presented with local recurrence and metastasis to the lung within two years after excision of mass. 


\section{Conclusion}

Leiomyoma and undifferentiated pleomorphic sarcoma are rare and to be considered in the differential diagnosis of spindle cell tumors of paratesticular region in view of treatment and prognosis.

\section{Reference}

1. Matias M, Carvalho M, Xavier L, Teixeira JA. Paratesticular sarcomas:two cases with different evolution. BMJ Case Rep 2014 Aug 21;2014.

2. Rana S, Sharma P, Singh P, Satarkar RN. Leiomyoma of scrotum:A rare case report. Iranian Journal of Pathology 2015;10(3):243-47.

3. Asotra S. Leiomyoma of scrotum. Arch Med Health Sci 2016;4(2):238-40.

4. Ulker V, Atalay HA, Cakir C, Sargan A. Giant malignant fibrous histiocytoma of the testis. Turk J Urol 2018;44(1):79-81.

5. Sherwani RK, Rahman K, Akhtar K, Zaheer S, Hassan MJ, Haider A. Leiomyoma of scrotum. Indian J Pathol Microbiol 2008;51(1):72-73.
6. Guo J, Zhou S, Rao NP, Pez GH. Pleomorphic malignant fibrous histiocytoma/ undifferentiated high grade pleomorphic sarcoma of the scrotum in a patient presenting as fournier gangrene: A case report. Appl Immunohistochem Mol Morphol 2010;18(5):473-78.

7. Takezawa K, Matsuoka Y, Takao T, Nonomura N, Tomita Y, Okuyama A. Myxofibrosarcoma of the spermatic cord: A case report. Nihon Hinyokika Gakkai Zasshi 2008;99(3):555-59.

8. Khandelwal A, Gupta SK, Singh M, Kumar V, Tiwari R, Singh A. High grade pleomorphic sarcoma of the scrotum:A rare clinical entity. UroToday Int J 2012;5(2):64-66.

9. Kallianpur AA, Kapali A, Shukla NK, Deo SV, Muduly D, Yadav R. Intrascrotal paratesticular malignant fibrous histiocytoma a rare case and its management. Indian $\mathrm{J}$ Cancer 2015;52(4):688-89.

10. Nascimento AF, Raut CP. Diagnosis and management of pleomorphic sarcomas (so-called 'MFH') in adults. J Surg Oncol 2008; 97(4):330-39.

*Corresponding author:

Meena N. Jadhav, Department of Pathology, Belagavi Institute of Medical Sciences Belagavi

Phone: +91 9886042408

Email: shubhamj2003@yahoo.co.in

Financial or other Competing Interests: None. 